\title{
Clinicopathological analysis of 12 patients with autoimmune pancreatitis
}

\author{
RUI ZHANG ${ }^{1}$, XIANG-DE SHI ${ }^{1}$, HONG ZENG $^{2}$, ZHAO-XI CAI $^{3}$, WEN-RUI WU ${ }^{1}$, \\ XIAN-HUAN YU ${ }^{1}$, JIE WANG ${ }^{1}$ and $\mathrm{CHAO} \mathrm{LIU}^{1}$ \\ ${ }^{1}$ Department Hepato-Pancreato-Biliary Surgery, Guangdong Provincial Key Laboratory of Malignant Tumor Epigenetics and \\ Gene Regulation; Departments of ${ }^{2}$ Pathology and ${ }^{3}$ Radiology, Sun Yat-Sen Memorial Hospital, \\ Sun Yat-Sen University, Guangzhou, Guangdong 510120, P.R. China
}

Received June 22, 2014; Accepted February 24, 2015

DOI: $10.3892 /$ etm.2015.2492

\begin{abstract}
Autoimmune pancreatitis (AIP) is a rare type of chronic pancreatitis that is often misdiagnosed as pancreatic cancer $(\mathrm{PaC})$. This study was undertaken to investigate the clinicopathological characteristics of AIP, in order to improve the diagnosis and treatment of the disease. Among the 271 patients with $\mathrm{PaC}$ who underwent pancreatoduodenectomy between January 2003 and December 2012 at the Sun Yat-Sen Memorial Hospital, chronic pancreatitis was identified and tissue samples obtained from 16 patients. The clinicopathological and imaging characteristics of 16 of the patients with chronic pancreatitis were analyzed retrospectively. The expression of immunoglobulin G4 (IgG4) in the pancreas tissue was detected by immunohistochemistry. Immunohistochemistry showed that IgG4 was highly expressed in 12 out of the 16 patients, and those 12 patients were diagnosed with AIP. Among those 12 patients, 6 presented with emaciation and 7 with jaundice and abdominal pain, respectively. Among the 16 included patients, 12 had an elevated level of serum $\gamma$-glutamyltransferase and 9 had an elevated level of serum carbohydrate antigen 19-9. The imaging features were as follows: Pancreatic enlargement in 11 patients (particularly pancreatic head enlargement), pancreatic miniature in 1, 'sausage-like' pancreatic changes in 4 and 'halo' sign pancreatic changes in 5. Massive plasma cell infiltration (11/12) and parenchymal fibrosis (8/12) were observed in the pancreatic tissues through pathology. These results suggest that combining imaging with IgG4 expression for the purpose of diagnosis can enhance the preoperative diagnostic value and reduce the rate of AIP misdiagnosis.
\end{abstract}

Correspondence to: Dr Chao Liu, Department of Hepato-PancreatoBiliary Surgery, Guangdong Provincial Key Laboratory of Malignant Tumor Epigenetics and Gene Regulation, 107 Yan-Jiang-Xi Road, Guangzhou, Guangdong 510120, P.R. China

E-mail: mdliuchao@hotmail.com

Key words: autoimmune pancreatitis, immunoglobulin G4, diagnosis, differentiation

\section{Introduction}

Autoimmune pancreatitis (AIP) is a type of chronic pancreas-specific inflammation mediated by an autoimmune inflammatory reaction (1). Yoshida et al (2) summarized its clinical features as follows: i) Increased serum $\gamma$-globulin or immunoglobulin G4 levels and presence of autoantibodies; ii) pancreatic enlargement; iii) occasional association with stenosis of the lower bile duct and other autoimmune diseases; iv) notable response to steroid therapy and v) histological findings of lymphoplasmacytic sclerosing pancreatitis (LPSP). Serum IgG4 levels have been frequently observed to be elevated in patients with AIP (3), indicating the presence of a systemic disease with abnormal IgG4 expression in the pancreas (4). The similar clinicopathological characteristics that AIP and $\mathrm{PaC}$ share often result in the misdiagnosis and unnecessary surgical treatment of patients with AIP (1). Since AIP responds markedly to steroid therapy, differentiating AIP from $\mathrm{PaC}$ is important in order to avoid unnecessary pancreatic resection. In the present study, the clinical data from 12 patients with AIP who were misdiagnosed with $\mathrm{PaC}$ were investigated for the purpose of improving the diagnosis and treatment of AIP.

\section{Patients and methods}

Patientsandpancreatictissuespecimens. Between January 2003 and December 2012, 271 patients with $\mathrm{PaC}$ underwent surgical treatment at the Department of Hepato-Pancreato-Biliary Surgery, Sun Yat-Sen Memorial Hospital, Sun Yat-Sen University (Guangzhou, China). Pancreatitis was identified histopathologically and pancreatic tissue specimens were obtained from 16 patients. The 16 patients were male with a mean age of $53.94 \pm 12.63$ years (range, 25-75 years). None of the patients had received chemotherapy or radiation therapy prior to the radical tumor resection. The paraffin-embedded pancreatic tissue specimens from each case were cut into $4-\mu \mathrm{m}$ sections consecutively. Routine histological examination was performed with hematoxylin and eosin (HE) staining in the Department of Pathology. AIP was diagnosed based on the Asian Diagnostic Criteria for AIP (5).

The present study was approved by the Ethics Committee of the Sun Yat-Sen Memorial Hospital and is in accordance 
with the Declaration of Helsinki of 1975. Written informed consent was obtained from either the patients or their guardian.

Immunohistochemistry. The immunohistochemical staining of the tissue sections for IgG4, using anti-IgG4 antibodies (ab109493; Abcam, Cambridge, UK) was completed according to the instructions defined in the Power Vision Two-Step kit (ZSG, Beijing, China) at the Medical Research Center of Sun Yat-Sen Memorial Hospital.

The staining was independently evaluated by two investigators who were unaware of the clinical data. The immunostained sections were first scanned under a light microscope at low magnification (x40), and five non-overlapping fields were then examined at a final magnification of $\mathrm{x} 400$. The final results were calculated by dividing the rate of $\mathrm{IgG} 4^{+}$plasma cells in the 10 high-power fields (HPFs; magnification, x400) by 10 . The positive cell rate was divided into four grades: Score $0,0 \%$ per $1 \mathrm{HPF}$; score $1,20 \%$ per $1 \mathrm{HPF}$; score $2,20-50 \%$ per $1 \mathrm{HPF}$ and score $3, \geq 50 \%$ per 1 HPF (6). When the assessment of the two observers differed, agreement was reached by using a double-headed microscope. A negative result was defined as a score of 0 and a positive result as a score of 3 .

Pancreatic imaging. A single radiologist, blinded to the clinical diagnosis, examined computed tomography (CT) $(16 / 16,100 \%)$ or magnetic resonance imaging (MRI) $(6 / 16,37.5 \%)$ scans of the 16 patients with pancreatitis and recorded the presence or absence of the following pancreatic abnormalities: Presence of a low-density mass with or without pancreatic ductal dilatation; pancreatic duct cutoff; diffuse glandular enlargement without ductal dilatation ( $>4 \mathrm{~mm}$ in diameter) or cutoff; focal glandular enlargement without ductal dilatation or cutoff; and diffuse pancreatic atrophy.

Follow-up. The 16 patients with chronic pancreatitis were followed up for 12-138 months (mean, 45 months). At each follow-up visit, clinical and laboratory data were collected and an abdominal ultrasound or CT scan was performed. The deadline of follow-up was October 2013.

\section{Results}

IgG4 expression in pancreatic tissues. In the immunohistochemical study, positive staining of IgG4 proteins was observed in the plasma cells (Fig. 1). The results, as evaluated by the two investigators, were identical for all slides. High IgG4 expression (scores 2 or 3 ) was observed in 6 out of the 16 pancreatitis samples (37.5\%), while moderate IgG4 expression (score 1) was also observed in 6 out of the 16 pancreatitis samples $(37.5 \%)$; the combination of the staining and imaging results led to these 12 patients being diagnosed with IgG4-positive AIP. Four cases scored 0.

Clinicopathological data. All 12 patients with AIP underwent pancreatoduodenectomy. The incidence rate of AIP was $4.43 \%$ (12/271). The general characteristics of these patients are summarized in Table I. Six patients presented with emaciation $(6 / 12,50.0 \%), 7$ with jaundice $(7 / 12,58.3 \%)$ and 7 with abdominal pain $(7 / 12,58.3 \%)$. Three patients had a history of diabetes, with 1 of them also suffering from chronic jaw gland inflammation. Of the included patients, 12 patients had an elevated level of $\gamma$-glutamyltransferase (>40 IU/1) and 9 had an elevated level of carbohydrate antigen 19-9 (CA19-9) (>37 and <200 U/ml) preoperatively; however, the blood and urine amylase levels of the 12 patients were normal. One patient exhibited no autoantibodies, while the other 11 patients were not examined for the presence of autoantibodies due to discharge from the hospital. The 12 patients with pathological confirmation of AIP all had resected specimens. The majority of the specimens were gray-yellow or gray-white, with either a fragile or a firm texture. HE staining showed microscopically detectable chronic pancreatitis: 8 specimens exhibited pancreatic fibrosis, 3 had pancreatic duct expansion, 11 exhibited plasma cell infiltration into the pancreatic tissues and 1 specimen did not show the inflammatory cell infiltration. The histological findings of the 12 cases of AIP indicated LPSP.

Imaging characteristics. A number of the CT and MRI findings of pancreatitis are presented in Table II and Fig. 2. Out of the 12 patients with AIP, 11 exhibited diffuse pancreatic enlargement and focal pancreatic enlargement in the head of the pancreas $(11 / 12,91.7 \%)$; 1 , pancreatic diminution (1/12, 8.3\%); 3, pancreatic duct expansion (3/12, 25.0\%); 4, 'sausage-like' pancreatic changes (4/12, 33.3\%); and 5, 'halo' sign pancreatic changes $(5 / 12,41.7 \%)$. During the scanning period of the CT imaging it was observed that the edges of the 12 pancreatic tissue specimens were flat and that the density of the specimens was uniform, without liquefaction necrosis or calcification. Through the enhanced scanning it was observed that the 12 pancreatic lesion areas were unequally intensified in a 'snowflake' pattern, and from the arterial- to the portal- and delayed-phase imaging, the pancreatic tissues were observed to exhibit progressively increased pancreatic lesion areas. Magnetic resonance cholangiopancreatography (MRCP) demonstrated that the major clinical manifestations of the patients were biliary obstruction, common bile duct, bile duct and gallbladder wall thickening. Four of the patients exhibited distal pancreatic duct expansion. No metastasis or infiltration of adjacent organs was identified in any of the 12 patients.

Prognosis and response to steroids. During the follow-up, 12 patients suffered from postoperative intermittent abdominal pain; 7 of these patients required treatment with painkillers. Following the surgery, 7 patients had an elevated level of total bilirubin. In 1 patient the level of serum IgG4 continuously declined for 11 months after he had received the metacortandracin hormone treatment $(0.6 \mathrm{mg} / \mathrm{kg})$, and the abnormal enlargement of the salivary gland was reversed.

\section{Discussion}

AIP is a type of chronic pancreas-specific inflammation caused by an autoimmune inflammatory reaction. Kasashima et al (7) proposed that AIP, apart from being a type of pancreatitis, was also a type of pancreatic injury occurring as a consequence of systemic disease. The manifestation of this injury as AIP results in it being easily misdiagnosed as $\mathrm{PaC}$. Thus, numerous 


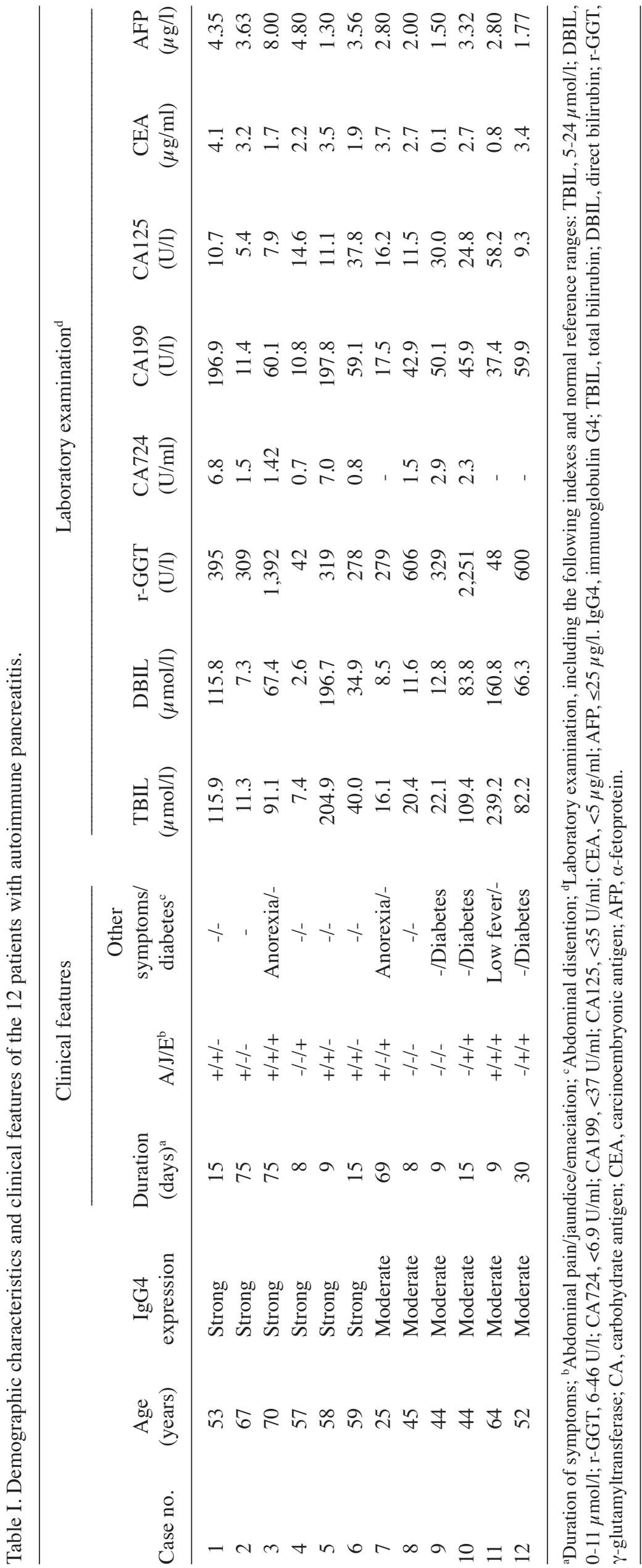



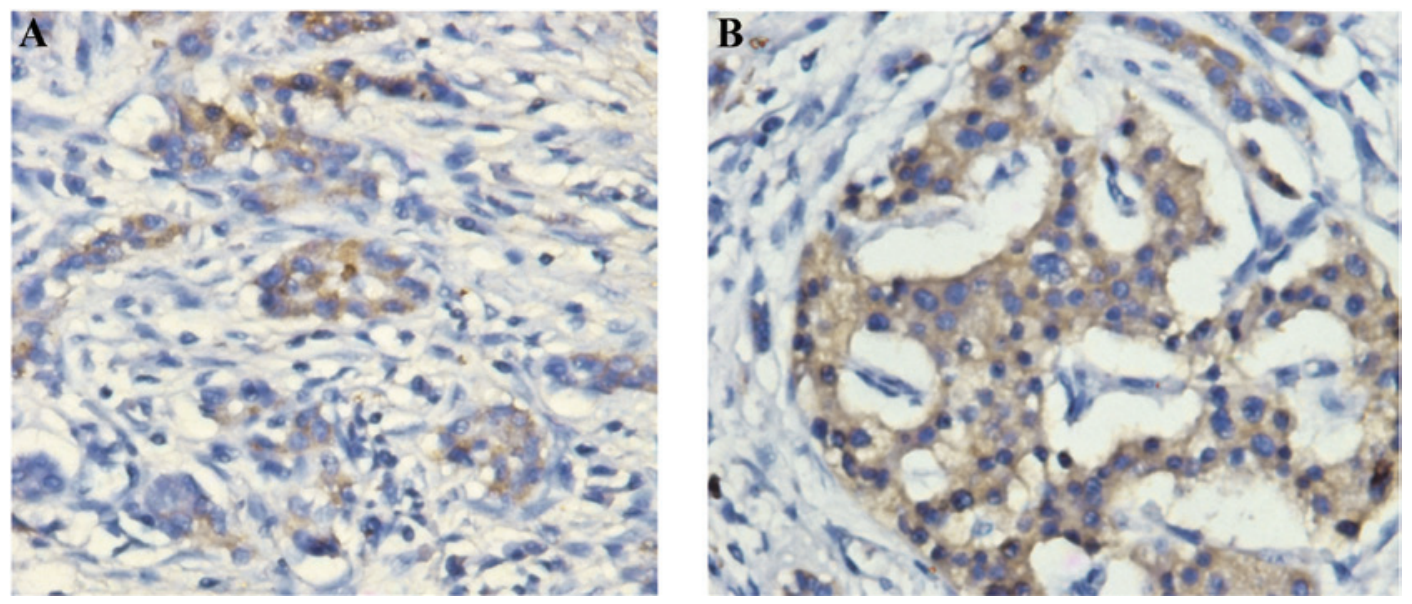

Figure 1. Immunohistochemical staining indicated that the plasma cells in the pancreas stained positive for immunoglobulin (Ig) G4 (Power Vision Two-Step method; magnification $\mathrm{x} 400$ ). (A) and (B) display two examples of tissues that stained positively for IgG4.
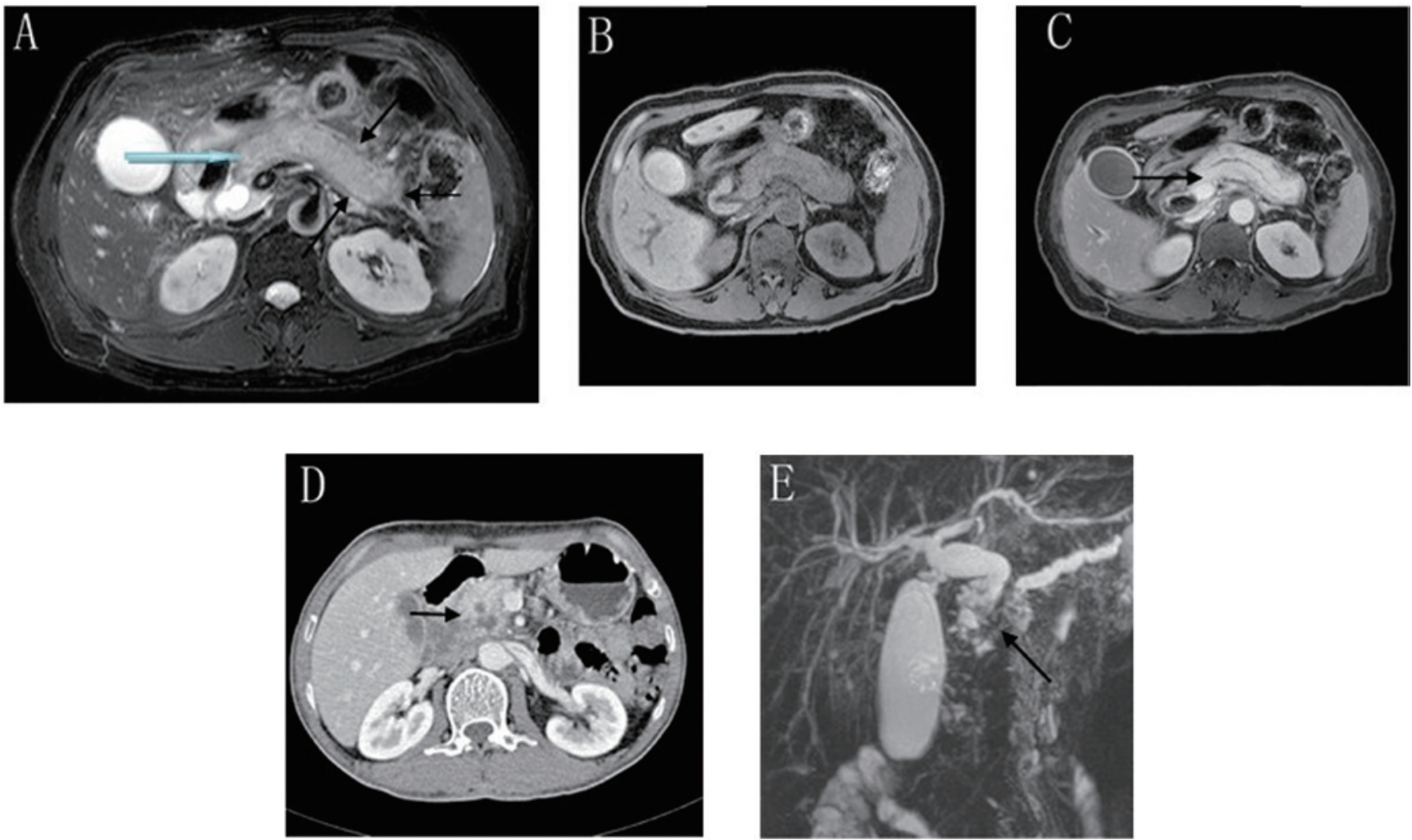

Figure 2. (A-D) Magnetic resonance imaging showed that (A) the T2W signal of the pancreas became higher, the pancreas underwent a 'sausage-like' change (blue arrow) and a bundle sheath-like low-signal envelope appeared around the pancreas (black arrow); (B) the pancreas T1W signal was reduced; (C) enhanced scanning revealed modality of the pancreas, the pancreatic duct swelling changed mildly (black arrow), with mild expansion, incrassation of the bile duct and chronic cholecystitis; (D) the pancreatic head enlarged (black arrow), the normal uncinate process forms had reduced with the uneven enlargement and lymph nodes were slightly increased. (E) Magnetic resonance cholangiopancreatography indicated that the lesions were primarily located in the pancreatic head, the distal bile duct exhibited stricture (black arrow) and the gallbladder pooled.

patients have undergone unnecessary surgical treatment. AIP and $\mathrm{PaC}$ have similar clinicopathological characteristics, such as abdominal pain, jaundice and weight loss (8). AIP typically occurs in patients $>55$ years old (9). The term 'AIP' is considered to encompass two different subtypes of the disease. The histological pattern of type 1 is known as LPSP, and is characterized by periductal lymphoplasmacytic infiltration, storiform fibrosis and obliterative venulitis $(10,11)$, while that of type 2 is known as idiopathic duct-centric pancre- atitis, which can be recognized by neutrophil infiltration and granulocytic epithelial lesions (12). All 12 cases in the present study were misdiagnosed preoperatively as $\mathrm{PaC}$, revealing the frequency of AIP misdiagnosis. The aim of the reflective analysis performed in this study was to facilitate the identification of and provide diagnostic strategies for AIP.

IgG4-positive plasma cell infiltration is widely considered to be the gold standard for AIP diagnosis (13), as was observed in the majority of the specimens collected in this study. It 
Table II. Imaging characteristics of the 16 patients.

Immunoglobulin G4

\begin{tabular}{|c|c|c|c|}
\hline \multirow[b]{2}{*}{ Computed tomography findings } & \multirow[b]{2}{*}{$\mathrm{N}$} & \multirow[b]{2}{*}{ Positive (n) } & \multirow[b]{2}{*}{ Negative (n) } \\
\hline & & & \\
\hline \multicolumn{4}{|l|}{ Diffuse enlargement of the pancreas } \\
\hline Yes & 11 & 11 & 0 \\
\hline No & 5 & 1 & 4 \\
\hline \multicolumn{4}{|c|}{ Focal enlargement in head of pancreas } \\
\hline Yes & 11 & 11 & 0 \\
\hline No & 5 & 1 & 4 \\
\hline \multicolumn{4}{|l|}{ Low-density mass } \\
\hline Yes & 7 & 7 & 0 \\
\hline No & 9 & 5 & 4 \\
\hline \multicolumn{4}{|l|}{ Diffuse pancreatic atrophy } \\
\hline Yes & 5 & 1 & 4 \\
\hline No & 11 & 11 & 0 \\
\hline \multicolumn{4}{|c|}{ Diffuse enlargement of the pancreas with ductal dilatation } \\
\hline Yes & 3 & 3 & 0 \\
\hline No & 9 & 9 & 0 \\
\hline \multicolumn{4}{|c|}{ Diffuse pancreatic atrophy with ductal dilatation } \\
\hline Yes & 0 & 0 & 0 \\
\hline No & 4 & 0 & 4 \\
\hline \multicolumn{4}{|l|}{ Capsule-like rim around pancreas } \\
\hline Yes & 0 & 0 & 0 \\
\hline No & 0 & 0 & 0 \\
\hline
\end{tabular}

was observed that the combination of moderate and higher IgG4 labeling of the plasma cells reached $100 \%(12 / 12)$ in the patients with AIP. In general, high IgG4 expression (scores 2 or 3) was observed in 6 out of the 16 pancreatitis samples, while moderate $\operatorname{IgG} 4$ expression was also found in 6 samples. Takahashi et al (13) reported that the accuracy of the spiral $\mathrm{CT}$ in the diagnosis of AIP is $68-76 \%$. The typical imaging finding in AIP is diffuse enlargement of the pancreas, i.e., the 'sausage-like' change. A well-defined capsule-like rim ('halo' sign), which is caused by fibrosis surrounding the lesions and can be observed in $12-40 \%$ of patients with AIP (14), is an important imaging characteristic of the disease and is rarely observed in malignant pancreatic tumors (15). In certain cases, a dilated main pancreatic duct can be noted through CT (16). Endoscopic retrograde cholangiopancreatography is widely used in Japan for the purpose of investigating obstructive jaundice and is mandatory in the Japanese criteria (17). MRCP has gained popularity for being a non-invasive method of obtaining high-quality images of the pancreaticobiliary tree (18). In the present study, 11 patients exhibited diffuse pancreatic enlargement and focal pancreatic enlargement in the head of pancreas $(11 / 12,91.7 \%), 1$ showed pancreatic diminution $(1 / 12,8.3 \%)$ and 3 had pancreatic duct expansion $(3 / 12,25.0 \%)$. During the scanning period, 12 pancreatic tissue specimens exhibited uniform density, without liquefaction necrosis and calcification. Enhanced scanning showed that the 12 pancreatic lesion areas were unequally intensified in a 'snowflake-like' shape. From the arterial phase to the portal phase, the pancreatic lesion area progressively increased. Four patients $(33.3 \%)$ exhibited pancreatic 'sausage-like' changes and 5 patients $(41.7 \%)$ experienced 'halo' sign changes. No metastasis or adjacent organ invasion, which can be used to differentiate AIP from $\mathrm{PaC}$ and facilitate its identification, was found in any of the 12 cases.

In the current study, 12 preoperative patients with AIP were misdiagnosed with $\mathrm{PaC}$ and underwent pancreatoduodenectomy, demonstrating that it was difficult to distinguish pancreatic cancer from AIP in the clinical setting; however, the serology, imaging and pathological features of AIP are unique. In terms of serology, the serum CA199 level of the 12 patients with AIP was $<200 \mathrm{U} / \mathrm{ml}$, while its level in patients with $\mathrm{PaC}$ is often $>200 \mathrm{U} / \mathrm{ml}$ (8). On the imaging level, 4 patients $(33.3 \%)$ exhibited pancreatic 'sausage-like' changes and 5 patients (41.7\%) showed 'halo' sign changes, while there was no evidence of metastasis or adjacent organ invasion. With regard to histology, 12 patients had a local stiffness of the pancreas, $11(91.7 \%)$ showed pancreatic plasma cell infiltration and $8(66.7 \%)$ showed pancreatic fibrosis. In terms of immunology, IgG4 expression was elevated in 12 out of $12(100 \%)$ patients with AIP; therefore, from a clinical point of view, patients with a preliminary diagnosis of $\mathrm{PaC}$, whose serum tumor markers are not high or who exhibit 'sausage-like' or 'halo' sign changes on imaging, without tumor invasion of adjacent organs, should be considered for a diagnosis of AIP. In such cases, the blood IgG4 levels of the patients should be measured. A serum IgG4 level of $1,350 \mathrm{mg} / \mathrm{l}$ has high sensitivity and specificity in the 
diagnosis of AIP (3). At present, glucocorticoids are considered to be highly efficacious in the long-term treatment of AIP (16). Prior to glucocorticoid treatment, it is necessary to completely rule out other types of pancreatic diseases. Clinical symptoms would improve significantly after 2-4 weeks through hormone therapy (19). In the present study, hormone therapy was recommended once patients were diagnosed with AIP. If the patients have only had a single surgery, symptoms such as abdominal pain and jaundice are unlikely to be relieved. Following the metacortandracin treatment, the level of IgG4 in 1 patient with AIP continuously declined and the abnormal enlargement of the salivary gland was reversed, thus proving that early glucocorticoid treatment can alleviate the clinical symptoms of the patient and improve the prognosis of AIP.

In conclusion, AIP is a rare type of pancreatitis. As the clinicopathological features of AIP are similar to those of $\mathrm{PaC}$, misdiagnoses of AIP as $\mathrm{PaC}$ are quite common, rendering unnecessary surgical resection. This study analyzed 12 patients with AIP in the Sun Yat-Sen Memorial Hospital. It was found that the serum CA199 levels in those patients were $<200 \mathrm{U} / \mathrm{ml}$. In imaging studies, pancreatic 'sausage-like' and 'halo' sign changes were observed, and the mass did not invade the adjacent organs and blood. From a histological perspective, plasma cells were found to have infiltrated widely. Immunology showed that cases with high levels of IgG4 were positive for $\mathrm{IgG} 4$ in biopsy. Combining medical imaging with IgG4 expression to obtain a diagnosis has proven to have significant practical value, which could reduce the rate of AIP misdiagnosis and the risk of unnecessary surgery.

\section{Acknowledgements}

This study was supported by The Special Research Foundation of the National Natural Science Foundation of China (no. 81301865).

\section{References}

1. Kamisawa T, Egawa N, Nakajima H, Tsuruta K, Okamoto A and Kamata N: Clinical difficulties in the differentiation of autoimmune pancreatitis and pancreatic carcinoma. Am J Gastroenterol 98: 2694-2699, 2003.

2. Yoshida K, Toki F, Takeuchi T, Watanabe S, Shiratori K and Hayashi N: Chronic pancreatitis caused by an autoimmune abnormality. Proposal of the concept of autoimmune pancreatitis Dig Dis Sci 40: 1561-1568, 1995.
3. Hamano H, Kawa S, Horiuchi A, et al: High serum IgG4 concentrations in patients with sclerosing pancreatitis. N Engl J Med 344: 732-738, 2001.

4. Kamisawa T, Takuma K, Egawa N, Tsuruta K and Sasaki T: Autoimmune pancreatitis and IgG4-related sclerosing disease. Nat Rev Gastroenterol Hepatol 7: 401-409, 2010.

5. Otsuki M, Chung JB, Okazaki K, et al; Research Committee of Intractable Pancreatic Diseases provided by the Ministry of Health, Labour and Welfare of Japan and the Korean Society of Pancreatobiliary Diseases: Asian diagnostic criteria for autoimmune pancreatitis: Consensus of the Japan-Korea Symposium on Autoimmune Pancreatitis. J Gastroenterol 43: 403-408, 2008.

6. Aoki S, Nakazawa T, Ohara H, et al: Immunohistochemical study of autoimmune pancreatitis using anti-IgG4 antibody and patients' sera. Histopathology 47: 147-158, 2005.

7. Kasashima S, Zen Y, Kawashima A, Endo M, Matsumoto Y and Kasashima F: A new clinicopathologic entity of IgG4-related inflammatory abdominal aortic aneurysm. J Vasc Surg 49: 1264-1271, 2009.

8. Ghazale A, Chari ST, Smyrk TC, et al: Value of serum IgG4 in the diagnosis of autoimmune pancreatitis and in distinguishing it from pancreatic cancer. Am J Gastroenterol 102: 1646-1653, 2007.

9. Zamboni G, Lüttges J, Capelli P, et al: Histopathological features of diagnostic and clinical relevance in autoimmune pancreatitis: a study on 53 resection specimens and 9 biopsy specimens. Virchows Arch 445: 552-563, 2004.

10. Park DH, Kim MH and Chari ST: Recent advances in autoimmune pancreatitis. Gut 58: 1680-1689, 2009.

11. Zhang L, Notohara K, Levy MJ, Chari ST and Smyrk TC: IgG4-positive plasma cell infiltration in the diagnosis of autoimmune pancreatitis. Mod Pathol 20: 23-28, 2007.

12. Zhang L, Chari S, Smyrk TC, et al: Autoimmune pancreatitis (AIP) type 1 and type 2: an international consensus study on histopathologic diagnostic criteria. Pancreas 40: 1172-1179, 2011.

13. Takahashi N, Fletcher JG, Fidler JL, Hough DM, Kawashima A and Chari ST: Dual-phase CT of autoimmune pancreatitis: A multireader study. AJR Am J Roentgenol 190: 280-286, 2008.

14. Kubota K, Iida H, Fujisawa T, et al: Clinical significance of swollen duodenal papilla in autoimmune pancreatitis. Pancreas 35: e51-e60, 2007.

15. Finkelberg DL, Sahani D, Deshpande V and Brugge WR: Autoimmune pancreatitis. N Engl J Med 355: 2670-2676, 2006.

16. Kamisawa T, Wakabayashi $\mathrm{T}$ and Sawabu N: Autoimmune pancreatitis in young patients. J Clin Gastroenterol 40: 847-850, 2006.

17. Okazaki K, Kawa S, Kamisawa T, et al; Research Committee of Intractable Diseases of the Pancreas: Clinical diagnostic criteria of autoimmune pancreatitis: Revised proposal. J Gastroenterol 41: 626-631, 2006.

18. Vaishali MD, Agarwal AK, Upadhyaya DN, Chauhan VS, Sharma OP and Shukla VK: Magnetic resonance cholangiopancreatography in obstructive jaundice. J Clin Gastroenterol 38: 887-890, 2004.

19. Chari ST, Takahashi N, Levy MJ, et al: A diagnostic strategy to distinguish autoimmune pancreatitis from pancreatic cancer. Clin Gastroenterol Hepatol 7: 1097-1103, 2009. 